\title{
Magnetic resonance imaging of experimental autoimmune encephalomyelitis in the common marmoset
}

\author{
Pietro Maggi ${ }^{a}$, Pascal Sati ${ }^{b}$, Luca Massacesi $^{\mathrm{c}}$ \\ a Department of Neurology, Hôpital Erasme-Université libre de Bruxelles, Belgium \\ b Translational Neuroradiology Unit, National Institute of Neurological Disorders and Stroke, National Institutes of Health, Bethesda, MD, USA \\ c Department of Neurosciences, Drug Research, and Child Health, University of Florence, Florence, Italy
}

\section{A R T I C L E I N F O}

Article history:

Received 14 April 2016

Accepted 28 September 2016

Available online $\mathrm{xxxx}$

\section{Keywords:}

Multiple sclerosis

Experimental autoimmune encephalomyelitis

Marmoset

Magnetic resonance imaging

Brain

Inflammation

Demyelination

\begin{abstract}
A B S T R A C T
Magnetic resonance imaging (MRI) is an invaluable tool for the diagnosis and monitoring of patients with multiple sclerosis (MS) as well as for the study of the disease pathophysiology. Because of its strong clinical, radiological and histopathological similarities with the human disease, experimental autoimmune encephalomyelitis (EAE) in the common marmoset has been studied more intensively over the past several years. Here, we review the current knowledge on MRI in the marmoset EAE, and we outline the physiopathological significance and translational values of these studies with respect to MS. Accumulating evidences suggest that the application of conventional, as well as non-conventional, MRI techniques in the marmoset EAE is a promising approach to elucidate the pathological processes underlying the development of inflammatory demyelinated lesions in the central nervous system, potentially improving the identification and development of new therapeutics.
\end{abstract}

(C) 2016 Published by Elsevier B.V.

\section{Introduction}

Multiple sclerosis (MS) is an immune-mediated disease of the central nervous system (CNS) pathologically characterized by the presence of focal demyelinated lesions in brain and spinal cord (Lassmann et al., 2007; Hohlfeld et al., 1995). Clinically, the most common form of MS begins with a relapsing remitting (RR) phase, characterized by neurological symptoms that partially or fully recover within days or weeks. Patients can remain in this phase for the whole life ( $20 \%$ cases, benign MS (Hawkins and McDonnell, 1999)) or switch to a progressive phase, named secondary progressive (SP) MS, characterized by accumulation of clinical disability over time. Although overt inflammation plays a major role in the first RR phase of the disease, the progressive phase seems mainly characterized by neurodegeneration with mostly compartmentalized inflammation (Absinta et al., 2015; Fischer et al., 2013).

At present, valid therapeutic options are available only for the RR phase of MS and no curative treatment exists. Indeed, the available treatments are more effective early in the course of the disease, when tissue damage and neurodegenerative processes are still minimal or absent. In order to further improve knowledge on the early treatable phase of the disease, recent research has been focusing on the sequence of events underlying the development of inflammatory lesions (Frohman et al., 2006). Although the "primum movens" determining these lesions to develop in the CNS is still largely unknown, an autoimmune reaction directed against CNS components, developing in a genetically and environmentally suitable background, is thought to be the base of this disease (t Hart and Massacesi, 2009). In this context, the close immunopathological similarities between the lesions observed in MS and in experimentally induced autoimmune encephalomyelitis (EAE) allowed to consider EAE a valid model of the effector pathogenic mechanisms of MS (t Hart and Massacesi, 2009).

\subsection{Advantages and limits of the EAE model}

The EAE model indeed is a well characterized model of CNS autoimmunity. The disease is induced by immunization of recipient animals with CNS antigens or myelin components together with an adjuvant, causing an autoimmune reaction against the CNS. EAE can be induced in a large variety of species (Baxter, 2007), but only a few forms of EAE are both clinically and pathologically close to MS. Indeed, the clinical and pathological features of this disease vary among the different species in which it was induced (mice, rats, rabbits, guinea pigs and monkeys) and among different models (based on the type of myelin antigen used to induce the disease), ranging from an acute monophasic disease to a relapsing-remitting one closer to human MS (t Hart and Massacesi, 2009).

Both in MS and EAE, the histopathology of the acute lesions, is characterized by inflammatory infiltrates, mostly lymphocytes and macrophages, cuffed around post capillary venules (Baxter, 2007). Despite a substantial histopathological similarity, the main difference between the two diseases is the artificial induction of autoimmunity in EAE whereas the causes of MS are less clear. Thus the first phase (afferent 
arm) of the immune response - that is, why and how the immune system begins to mount an autoimmune response against the CNS - definitively differs between the two diseases. Nonetheless, the close histopathological similarity between MS and EAE suggests that the second phase (the effector arm) of the immune response may be very similar (t Hart and Massacesi, 2009). In this contest EAE is widely considered a valid model to study the effector arm of MS pathogenesis. As a proof of relevance, three therapeutics commonly used to treat MS patients have been developed on the ground of EAE models: glatiramer acetate, mitoxantrone and natalizumab (Yednock et al., 1992). However, a large number of other potential therapies effective in mice EAE failed to have clinical efficacy in human MS (Sriram and Steiner, 2005; Steinman and Zamvil, 2006). This is likely to be due to the large evolutionary distance between rodents and humans. In addition, inbred laboratory mice lack a pathogen-educated immune system, an important discrepancy with the human disease where infections are thought to play a crucial role in pathogenesis (Lathrop et al., 2011). Lesions in the mice EAE model are mostly confined to the brain stem and spinal cord and the pathology often does not includes demyelination (Hauser, 2015). Moreover, the small size of the mice brain makes them unsuitable for in vivo MRI studies (Jordan et al., 1999; Hart et al., 1998). Therefore the development of an EAE model in larger animals, with a closer evolutionary relationship to human, allowed to bridge the gap between mice EAE and human MS (Kap et al., 2010).

\subsection{EAE in the common marmoset}

Nonhuman primates are genetically, immunologically, and microbiologically closer to humans, and for this reason EAE has been developed in old and new world monkeys. EAE in macaques (old world monkeys) is characterized by an hyperacute destructive CNS disease usually causing the death of the animal within a few days after onset ( $t$ Hart and Massacesi, 2009; Kap et al., 2010; t Hart et al., 2000), thus making this model closer to acute disseminated encephalo-myelitis than to MS. On the other hand, EAE in the common marmoset (new world monkey) creates a CNS disease very similar to MS. The common marmoset (Callitrix jacchus) is a small monkey with an adult weight of 300$400 \mathrm{~g}$ ( $\mathrm{t}$ Hart et al., 2000). Female marmosets commonly give birth to two non-identical twins. These twins are bone marrow chimeras, a feature that allows transfer of immune cells between twins, even when not identical, without inducing rejection (Genain and Hauser, 1996). Two main marmoset EAE models have been developed so far. The first model, originally described by Massacesi et al., is induced with white matter (WM) homogenate (WMH) (Massacesi et al., 1995) giving perivenular brain and spinal cord WM lesions ( $t$ Hart and Massacesi, 2009; Gaitan et al., 2013) pathologically resembling the lesions observed in MS autopsies. The second model, induced with recombinant human myelin oligodendrocite glycoprotein (rhMOG) gives generally fewer but larger demyelinated lesions. Although the rhMOG model recapitulates many of the pathological and immunological aspects of human MS (tHart et al., 2015), in our experience the disease in this model appears more aggressive, sometimes hemorrhagic, and therefore less close to MS than the former one (Gaitan et al., 2013).

An important difference between the marmosets and the pathogenfree laboratory rodents is the natural exposure to environmental infections. As it happens in humans, the development of the marmoset's immune system is influenced by environmental pathogens and chronic latent infections (tHart et al., 2015; Leibovitch et al., 2013). It has been hypothesized that through a mechanism of brain-pathogen antigen cross-recognition and/or direct cellular infection, these environmental pathogens play an important role in the development of autoimmunity both in MS and in EAE (Berer et al., 2011). Being genetically, immunologically and environmentally closer to humans compared to laboratory mice, EAE in the marmosets can well recapitulate the most important MS susceptibility factors: genes and the environment. Additionally, the relatively small size of these primates and the higher white-to- gray matter ratio compared to rodent brains make them highly suitable to study in vivo the development of white matter brain lesions by magnetic resonance imaging (MRI).

\subsection{Magnetic resonance imaging in marmoset $E A E$}

MR imaging is an essential tool for the diagnosis and monitoring of patients with MS, but it can also provide a window into the disease pathogenesis. However, the underlying biology of the MRI findings is often difficult to establish in human studies. This issue can be overcome with animal models which enable a direct comparison of MR images acquired in vivo with histopathology performed post mortem. Another advantage of animal models is the possibility to obtain pre-morbid MR images and to follow the development of brain lesions from the time of their first appearance until the termination of the experiment (Gaitan et al., 2013; Maggi et al., 2014a). Similarly to human studies, conventional and non-conventional MRI techniques can be applied to these animals, thus allowing to better understand the biological correlates of the MRI findings, as well as validating novel more specific MRI markers of the different damages (such as demyelination, axonal pathology, iron deposition) occurring in brain lesions.

\subsection{Conventional MRI techniques}

In MS, conventional MRI techniques such as T2-weighted (T2w), proton-density weighted (PDw) and T1-weighted ( $\mathrm{T} 1 \mathrm{w})$ sequences are commonly used to follow the development of new brain lesions over time as well as to evaluate the evolution of older lesions (Polman et al., 2011). The same techniques can be applied to the marmoset EAE at various magnetic field strengths (1.5 T, $3 \mathrm{~T}$ and $7 \mathrm{~T}$ ) (Jordan et al., 1999; Gaitan et al., 2013; Maggi et al., 2014a; Blezer et al., 2007; Helms et al., 2013). Similarly to MS, new WM lesions in the marmoset EAE appear hyperintense on T2w and PDw and hypointense on T1w sequences. The hyperintense signal in both T2w and PDw is nonspecific, and can be correlated with both inflammation and tissue damage including demyelination. Within a few weeks after lesion appearance, the hyperintense focal area tends to reduce in size, possibly because of reabsorption of perilesional edema ( $t$ Hart and Massacesi, 2009). In the marmoset EAE, this shrinkage of the T2w hyperintense area also correlates with partial remyelination (Maggi et al., 2014a). Longitudinal MRI of the marmoset EAE demonstrates that new lesions develop over time and can form in any of the functional systems of the CNS, thus reproducing the classic pattern of lesion development in MS with both dissemination in time and space ( $\mathrm{t}$ Hart and Massacesi, 2009; Jordan et al., 1999).

The different locations of EAE lesions in the brain (periventricular, juxtacortical, infratentorial) reproduce MS lesions ( $t$ Hart and Massacesi, 2009) [Fig. 1]. Moreover, EAE lesions tend to develop around parenchymal veins, similarly to MS (Adams and Kubik, 1952). This was recently demonstrated using susceptibility-based imaging, also called $\mathrm{T} 2 *$-weighted $(\mathrm{T} 2 * \mathrm{w})$ imaging, which is particularly sensitive to the magnetic susceptibility of venous blood (Sati et al., 2014; Maggi et al., 2014b). On T2*w images, EAE lesions in the white matter appear as focal hyperintensities with a hypointense line (central vein) running through them (Sati et al., 2014) [Fig. 2]. Availability of T2*w images before EAE induction, as well as frequent $\mathrm{T}^{*} \mathrm{w}$ imaging following induction, allowed to demonstrate that EAE lesions develop around preexisting parenchymal veins (Gaitan et al., 2013).

Similarly to MS, gray matter (GM) pathology has also been reported in the marmoset EAE (Pomeroy et al., 2005). Although deep GM lesions can be observed by MRI in marmoset EAE [Fig. 3], cortical GM lesions remain difficult to detect in vivo, as thus far leukocortical lesions only have been observed [Fig. 3]. The poor detection by MRI of intracortical and subpial lesions is most likely due to the relative paucity of myelin in the cortex (Ge, 2006), as well as the lower amount of edema in those types of lesions (Lucchinetti et al., 2011). 


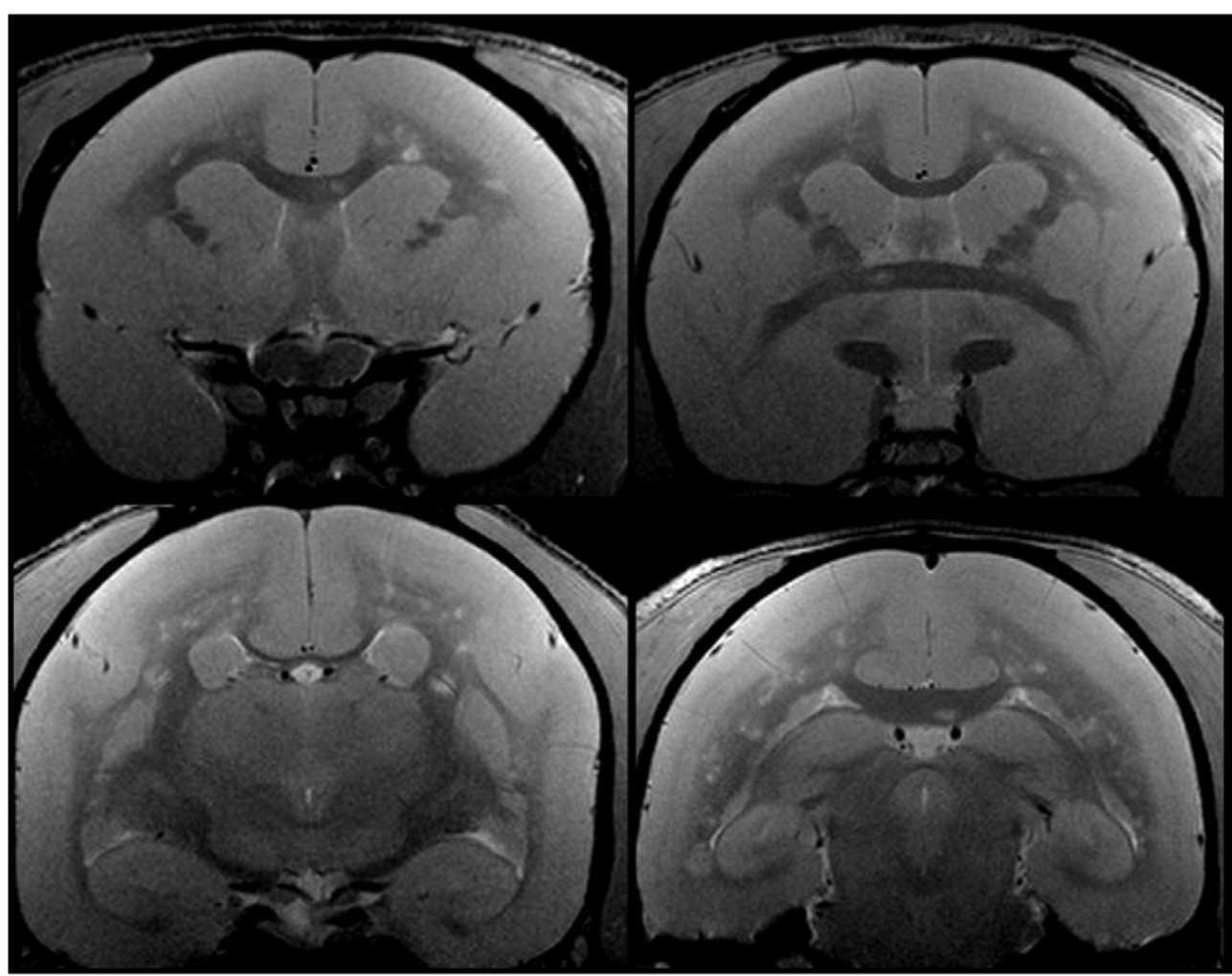

Fig. 1. Proton density-weighted images collected on a marmoset EAE showing the distribution of hyperintense lesions throughout the cerebral white matter.

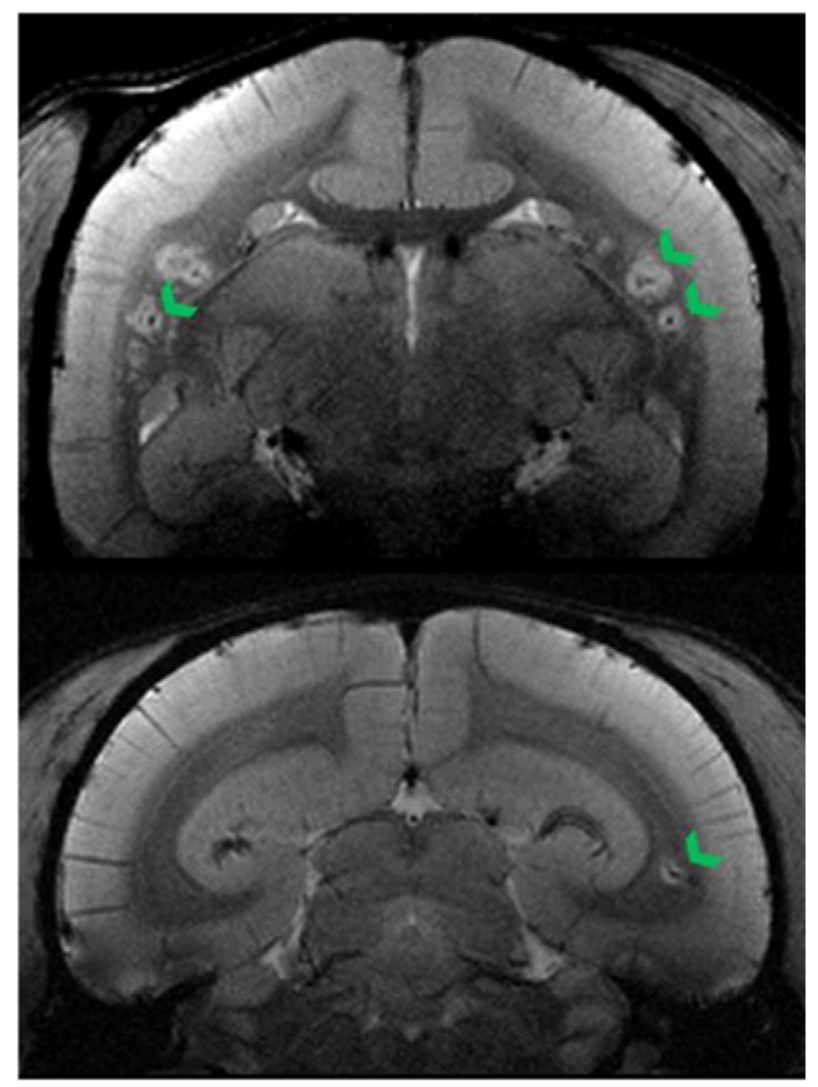

Fig. 2. T2*-weighted images collected on a marmoset EAE showing the perivenular distribution of white matter lesions (green arrows). (For interpretation of the references to color in this figure legend, the reader is referred to the web version of this article.)
Another common feature between MS and EAE lesions is related to the integrity of the blood brain barrier (BBB) during the lesion development. Similarly to MS lesions, the status of the BBB can be investigated by MRI in marmoset EAE with the injection of gadolinium-based contrast agent (GBCA). When the BBB of newly formed lesions is leaking, GBCA can penetrate the brain parenchyma and modifies the magnetic properties of the brain tissue (GBCA shortens the longitudinal relaxation time (T1) of the tissue (Soon et al., 2007). This usually results in an increase of signal intensity in the lesion area which is clearly visible on T1w images acquired post- GBCA injection (Hart et al., 1998) [Fig. 4]. Note that older (although still inflamed) lesions remain usually hypo- or isointense on post-GBCA T1w images due to a closed BBB (our data, unpublished).

\subsection{Nonconventional MRI techniques}

Over the past decade, various nonconventional MRI techniques have been developed to provide more specificity and/or more sensitivity toward the pathological substrates of MS lesions. Some of these advanced techniques (such as magnetization transfer imaging and T1 relaxometry) have also been applied to the marmoset EAE (Maggi et al., 2014a; Blezer et al., 2007; Richards et al., 1995).

For example, magnetization transfer (MT) imaging is a semiquantitative technique that is sensitive to change in myelin content in the CNS. A magnetization transfer ratio (MTR) image is generated from the relative difference between two gradient-echo images acquired with and without an off-resonance saturation pulse. Because myelin is a lipid bilayer with a high macromolecule density, white matter will be more affected by the MT-saturation pulse, resulting in a higher MTR value than for GM or CSF [Fig. 5]. In acute EAE lesions, a decrease in MTR has been associated with inflammation (Blezer et al., 2007; t Hart et al., 2004) most likely due to the increased water content from edema [Fig. 5]. Interestingly, a decrease in MTR was also reported in normal-appearing white matter preceding MS lesion formation (Filippi et al., 1998; Wuerfel et al., 2004). This MTR change may be 


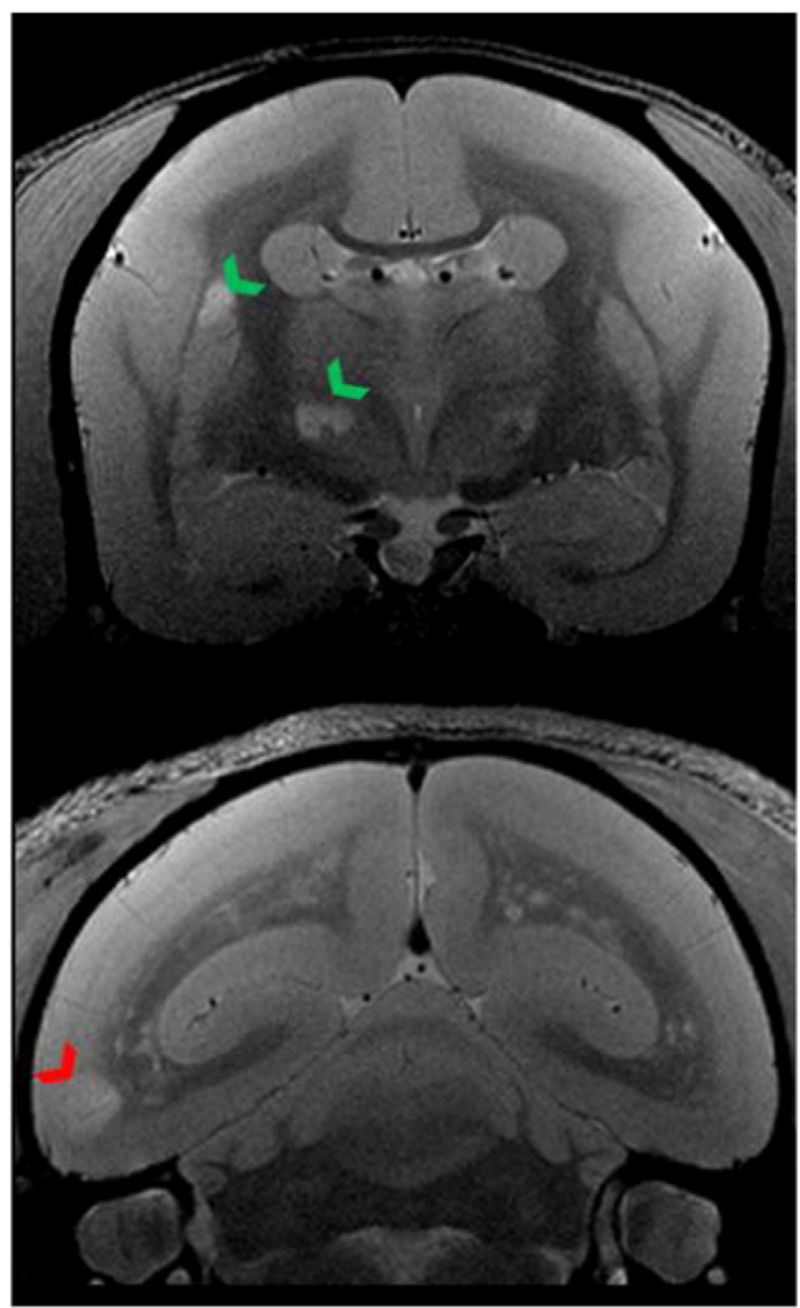

Fig. 3. Proton density-weighted images collected on a marmoset EAE showing deep gray matter lesions (green arrows) and a leukocortical lesion (red arrow). (For interpretation of the references to color in this figure legend, the reader is referred to the web version of this article.)

related to a subtle increase in BBB permeability occurring at the earliest stages of lesion formation. This hypothesis was recently investigated in the marmoset EAE using T1 relaxometry. Indeed, by acquiring T1 maps before and after injection of GBCA, permeability of the BBB can be evaluated by the change in $\mathrm{R} 1$ (=1/T1) relaxation rate (Soon et al., 2007). Analyzing 189 lesions from five different EAE animals, it was found that the BBB becomes focally and increasingly permeable starting up to one month before the development of WM lesions (Maggi et al., 2014a).

\subsection{Comparison of MRI findings with histopathology}

Pioneer studies investigating MRI lesions in the marmoset EAE (Jordan et al., 1999)demonstrated that these are very similar to MS lesions visualized by MRI ( $t$ Hart and Massacesi, 2009). The characterization of the pathology underlying different types of marmoset EAE brain MRI lesions allowed for the first time to characterize also the heterogeneous repertoire of the different immune-pathological substrates of the corresponding brain MRI lesions observed in MS. Noteworthy virtually all the different MRI lesions described in MS could also be observed and pathologically characterized in the marmoset EAE model (Hart et al., 1998; Maggi et al., 2014a). Exactly as in MS early marmoset EAE lesions are demyelinated, can occur both in WM and GM and show Dawson's finger like site and shape, tending to develop around parenchymal veins, (Adams and Kubik, 1952; JM., 1868). A classic feature of early MS lesions, commonly known as acute lesions, is the presence of a cuff of inflammatory cells (mostly lymphocytes and dendritic cells) around the central vein (Prineas and Parratt, 2012). This is also observed in marmoset EAE (Jordan et al., 1999), where acute lesions are associated with profound leakage of the blood brain barrier (BBB) allowing inflammatory cells and factors to enter the lesion parenchyma (Hart et al., 1998; Maggi et al., 2014a; Katz et al., 1993). In the parenchyma surrounding the central inflamed blood vessel, myelin is usually damaged and picked up from large activated macrophages, exactly as in MS (Hart et al., 1998; Maggi et al., 2014a; Prineas and Parratt, 2012). Both in MS and marmoset EAE (Jordan et al., 1999) new inflammatory lesions detected from Gd based MRI, are mainly associated to extensive demyelination with relative sparing of the axons, whereas older lesions are characterized by markedly reduced inflammatory activity associated to various degree of axonal damage (Hauser, 2015; Jordan et al., 1999; Hart et al., 1998). Both in MS and marmoset EAE inflammatory lesions not only T lymphocytes but also B cells are found (tHart et al., 2015; Maggi et al., 2014a). In the animal model Genain et al. showed that antibodies and presumably B cells are required for the development of demyelination (Genain et al., 1996; Genain et al., 1995). This is particularly relevant considering the increasing recognition that $\mathrm{B}$ cells are gaining in MS pathogenesis and therapy development (Absinta et al., 2015; Hauser, 2015). As in MS, also in marmoset EAE (Pomeroy et al., 2005), the nature of both GM and WM lesions is inflammatory but WM lesions only are commonly associated with leakage of the BBB (Lucchinetti et al., 2011). Noteworthy investigation of the pathological substrates of brain MRI finding is increasingly gaining importance in experimental neurosciences. For improving rapidity and precision of this kind of investigation both in MS and marmoset EAE brains, a novel simple method has recently been developed (Guy

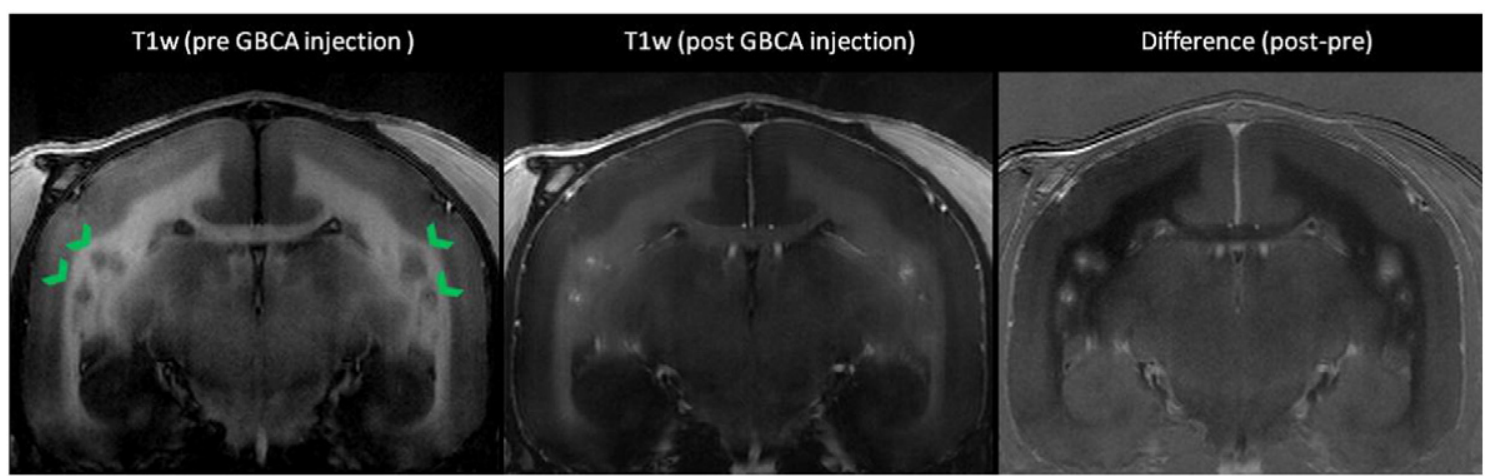

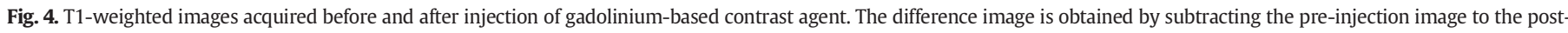

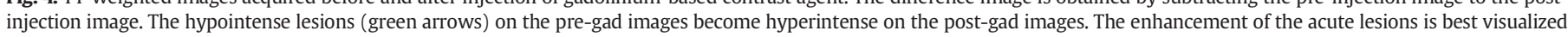
on the difference image. (For interpretation of the references to color in this figure legend, the reader is referred to the web version of this article.) 


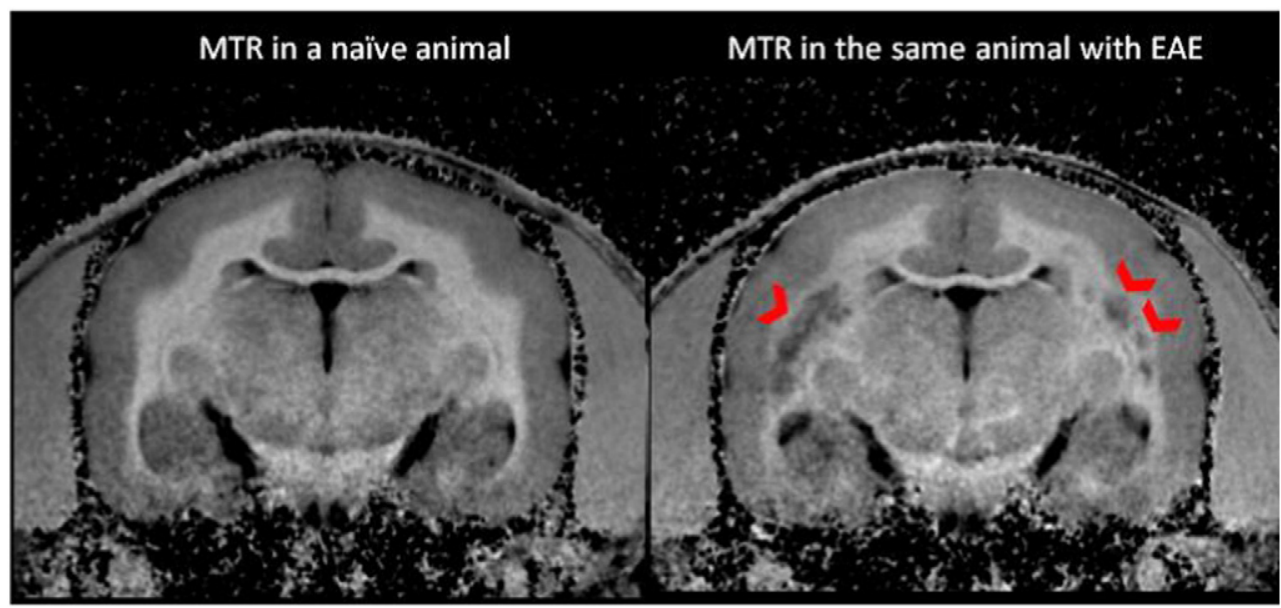

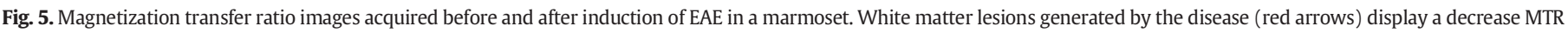
most likely due to edema and/or demyelination. (For interpretation of the references to color in this figure legend, the reader is referred to the web version of this article.)

et al., 2015; Absinta et al., 2014). This method relies on custom-built 3D printed brain holders to maintain the same brain orientation applied to the in vivo MRI acquisition and the sectioning of histological slabs [Fig. 6]. This method reduces the burden of manually matching MRI and pathology images at a relatively low monetary cost.

\subsection{Lesion histopathology as a function of lesion age}

For the histopathological characterization of the lesion evolution process over time, serial in vivo MRI can be used to provide information regarding the precise age of the lesions. Recent data on newly forming lesions in MS (Gaitan et al., 2011; Absinta et al., 2013) have shown how the dynamic pattern of contrast enhancement can give specific information about the age of a lesion, a parameter that in human MS autopsy studies it is difficult to directly establish. Indeed the time frame between the first appearance of a lesion (potentially detectable with in vivo brain imaging) and the death of the patient is usually determined only indirectly, by the quality of myelin degradation products present within macrophages' and/or the expression of macrophage activation markers (Bruck et al., 1995). Although this same approach has been successfully applied to marmoset histopathology by t'Hart and colleagues ( $t$ Hart and Massacesi, 2009), we recently showed a strategy to in vivo precisely determine the age of a lesion in the marmoset EAE model, by comparing the post mortem histopathology with the in vivo imaging. As explained before in this paper, the acquisition of a serial highly frequent in vivo data set and the availability of pre-morbid images, allows precise determination (in the order of days) of a lesion age (Maggi et al., 2014a). Thus the entire process of lesion development from its perivenular onset to its centrifugal expansion and, finally, its shrinkage and repair, can be tracked over time. Consistently with the in vivo T2* imaging results of Gaitan et al. (Gaitan et al., 2013), lesions younger than one week were found to develop around veins with a lymphocytic perivascular cuff surrounded by demyelination and by large activated blood derived macrophages in the parenchima. These lesions appear on T2-w images as focal hyperintensities in the brain WM and are associated with elevated levels of BBB permeability as shown from pre and post Gd T1 mapping. Interestingly, these early lesions resemble the "phagocytic areas" described in MS pathology (Henderson et al., 2009). Over the weeks, like in MS, we assist to an enlargement of the initial area of T2-w signal hyperintesity, as the process of inflammatory demyelination and its attendant activated blood derived macrophages infiltration moves outward toward the lesion edges (Gaitan et al., 2011; Henderson et al., 2009). Lesions older than five weeks appear as small areas of residual T2-w hyperintensity on MRI. These lesions shrink over time and are associated to reduced levels of BBB permeability, suggesting a reabsorption of the inflammatory oedema and repair. Indeed, inflammatory cells and active demyelination are markedly reduced in these lesions featuring pale myelin at the lesion borders, a pattern that in MS has been related to remyelination (Lassmann, 2011). Pre and post Gd T1 mapping MRI applied serially to the marmoset EAE brain shows how the BBB becomes focally and increasingly permeable starting up to one month before the appearance of $\mathrm{T} 2 \mathrm{w}$ demyelinated

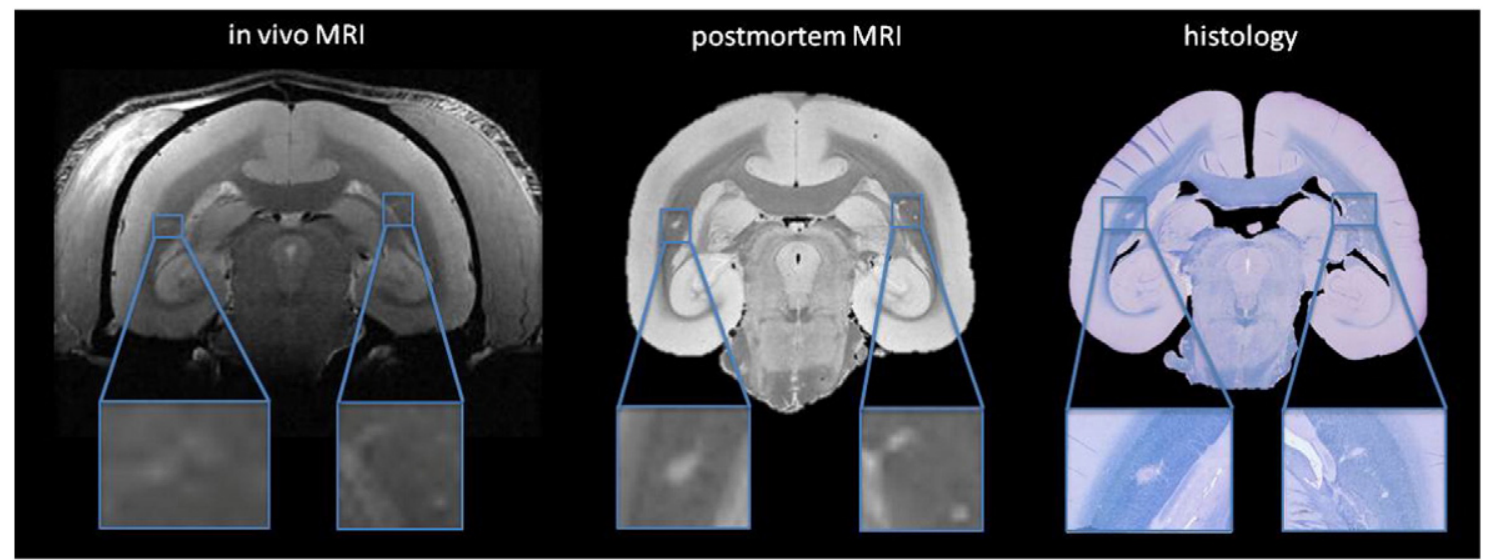

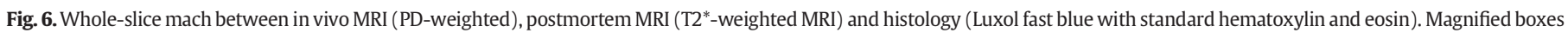

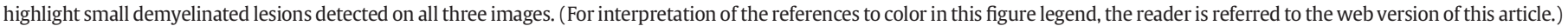


lesions. These early BBB permeability changes observed in this study corresponded on the histopathology, to small foci of perivascular lymphocytes and microglia activation without demyelination nor blood derived macrophages infiltration (Maggi et al., 2014a).

In human MS studies, the pathological correlates of the prelesional MRI changes detected by longitudinal nonconventional MRI are difficult to elucidate. Indeed, areas of future lesion development or prelesions are rarely identified in MS biopsy samples due to the chronic nature of the disease, with little new lesion formation later in the course of the disease, and to the fact that acute MS lesions are only rarely fatal (Barnett and Prineas, 2004). In the few histopathological studies available on MS early lesions, small areas of lymphocites perivascular cuffing and parenchymal microglia activation have been hypothetized to be areas of future lesion formation (Henderson et al., 2009). In these early lesions BBB opening and fibrinogen leakage have been shown to be crucial determinants for the activation of adaptive and innate immune responses (Marik et al., 2007) (Davalos et al., 2012) and for the development of autoimmunity in the CNS (Ryu et al., 2015). Similar early lesions in the marmoset EAE feature indistinguishable rate of BBB permeability increase over time compared to areas evolving in demyelinated lesions. Thus, lymphocyte perivascular cuffing and microglia activation appears to be the forerunners of focal lesions and to precede demyelination (Maggi et al., 2014a). Considering the high histopathological similarity between marmoset EAE and human MS, this finding may have an important translational value with respect to MS.

\section{Concluding remarks}

MRI imaging is believed to be a valid tool to perform an in vivo estimation of brain pathology, avoiding in many cases the requirement of brain biopsy, a more invasive and morbidity associated technique (Auriel et al., 2015). MRI in the marmoset EAE gives the unique opportunity of applying the same imaging protocols generally adopted in the clinical practice to an experimental model strongly resembling the human disease, thus shedding light on the histopathological correlates of the MR findings and therefore of the disease. Moreover in the marmoset EAE, not only conventional but also non-conventional MRI techniques can be obtained at ultra-high field strength with important implications for the development of new imaging markers of disease. In addition availability in the marmoset EAE of premorbid images, of longitudinal MRI protocols as well as of prompt imaginghistopathology correlations, allow to elucidate the pathological processes underlying the development of lesions in the CNS. Finally, given the strong environmental, immunological and histopathological similarities between the two diseases, results obtained in the marmoset can be reliably translated to the human disease helping to elucidate the complex pathogenesis of MS and improving the identification and development of new therapeutics.

\section{References}

Absinta, M., Nair, G., Filippi, M., Ray-Chaudhury, A., Reyes-Mantilla, M.I., Pardo, C.A., et al., 2014. Postmortem magnetic resonance imaging to guide the pathologic cut: individualized, 3-dimensionally printed cutting boxes for fixed brains. J. Neuropathol. Exp. Neurol. 73 (8), 780-788.

Absinta, M., Sati, P., Gaitan, M.I., Maggi, P., Cortese, I.C., Filippi, M., et al., 2013. Seven-tesla phase imaging of acute multiple sclerosis lesions: a new window into the inflammatory process. Ann. Neurol.

Absinta, M., Vuolo, L., Rao, A., Nair, G., Sati, P., Cortese, I.C., et al., 2015. Gadolinium-based MRI characterization of leptomeningeal inflammation in multiple sclerosis. Neurology 85 (1), 18-28

Adams, R.D., Kubik, C.S., 1952. The morbid anatomy of the demyelinative disease. Am. J. Med. 12 (5), 510-546.

Auriel, E., Charidimou, A., Gurol, M.E., Ni, J., Van Etten, E.S., Martinez-Ramirez, S., et al., 2015. Validation of clinicoradiological criteria for the diagnosis of cerebral amyloid angiopathy-related inflammation. JAMA Neurol. 1-6.

Barnett, M.H., Prineas, J.W., 2004. Relapsing and remitting multiple sclerosis: pathology of the newly forming lesion. Ann. Neurol. 55 (4), 458-468.

Baxter, A.G., 2007. The origin and application of experimental autoimmune encephalomyelitis. Nat. Rev. Immunol. 7 (11), 904-912.
Berer, K., Mues, M., Koutrolos, M., Rasbi, Z.A., Boziki, M., Johner, C., et al., 2011. Commensal microbiota and myelin autoantigen cooperate to trigger autoimmune demyelination. Nature 479 (7374), 538-541.

Blezer, E.L., Bauer, J., HP, B., Nicolay, K., t Hart, B.A., 2007. Quantitative MRI-pathology correlations of brain white matter lesions developing in a non-human primate model of multiple sclerosis. NMR Biomed. 20 (2), 90-103.

Bruck, W., Porada, P., Poser, S., Rieckmann, P., Hanefeld, F., Kretzschmar, H.A., et al., 1995 Monocyte/macrophage differentiation in early multiple sclerosis lesions. Ann. Neurol. 38 (5), 788-796.

Davalos, D., Ryu, J.K., Merlini, M., Baeten, K.M., Le Moan, N., Petersen, M.A., et al., 2012 Fibrinogen-induced perivascular microglial clustering is required for the development of axonal damage in neuroinflammation. Nat. Commun. 3, 1227.

Filippi, M., Rocca, M.A., Martino, G., Horsfield, M.A., Comi, G., 1998. Magnetization transfer changes in the normal appearing white matter precede the appearance of enhancing lesions in patients with multiple sclerosis. Ann. Neurol. 43 (6), 809-814.

Fischer, M.T., Wimmer, I., Höftberger, R., Gerlach, S., Haider, L., Zrzavy, T., et al., 2013. Disease-specific molecular events in cortical multiple sclerosis lesions. Brain 136 (6), 1799-1815.

Frohman, E.M., Racke, M.K., Raine, C.S., 2006. Multiple sclerosis-the plaque and its pathogenesis. N. Engl. J. Med. 354 (9), 942-955.

Gaitan, M.I., Maggi, P., Wohler, J., Leibovitch, E., Sati, P., Calandri, I.L., et al., 2013. Perivenular brain lesions in a primate multiple sclerosis model at 7-tesla magnetic resonance imaging. Mult. Scler.

Gaitan, M.I., Shea, C.D., Evangelou, I.E., Stone, R.D., Fenton, K.M., Bielekova, B., et al., 2011 Evolution of the blood-brain barrier in newly forming multiple sclerosis lesions. Ann. Neurol. 70 (1), 22-29.

Ge, Y., 2006. Multiple sclerosis: the role of MR imaging. AJNR Am. J. Neuroradiol. 27 (6), 1165-1176.

Genain, C.P., Hauser, S.L., 1996. Allergic encephalomyelitis in common marmosets: pathogenesis of a multiple sclerosis-like lesion. Methods (San Diego, Calif). 10 (3), 420-434.

Genain, C.P., Abel, K., Belmar, N., Villinger, F., Rosenberg, D.P., Linington, C., et al., 1996 Late complications of immune deviation therapy in a nonhuman primate. Science (New York, NY) 274 (5295), 2054-2057.

Genain, C.P., Nguyen, M.H., Letvin, N.L., Pearl, R., Davis, R.L., Adelman, M., et al., 1995. Antibody facilitation of multiple sclerosis-like lesions in a nonhuman primate. J. Clin. Invest. 96 (6), 2966-2974

Guy, J.R., Sati, P., Leibovitch, E., Jacobson, S., Silva, A.C., Reich, D.S., 2015. Custom fit 3Dprinted brain holders for comparison of histology with MRI in marmosets. J. Neurosci. Methods 257, 55-63.

Hart, B.A., Bauer, J., Muller, H.J., Melchers, B., Nicolay, K., Brok, H., et al., 1998. Histopathological characterization of magnetic resonance imaging-detectable brain white matter lesions in a primate model of multiple sclerosis: a correlative study in the experimental autoimmune encephalomyelitis model in common marmosets (Callithrix jacchus). Am. J. Pathol. 153 (2), 649-663.

Hauser, S.L., 2015. The Charcot lecture | beating MS: a story of B cells, with twists and turns. Mult. Scler. 21 (1), 8-21.

Hawkins, S.A., McDonnell, G.V., 1999. Benign multiple sclerosis? Clinical course, long term follow up, and assessment of prognostic factors. J. Neurol. Neurosurg. Psychiatry 67 (2), 148-152.

Helms, G., Garea-Rodriguez, E., Schlumbohm, C., Konig, J., Dechent, P., Fuchs, E., et al. 2013. Structural and quantitative neuroimaging of the common marmoset monkey using a clinical MRI system. J. Neurosci. Methods 215 (1), 121-131.

Henderson, A.P., Barnett, M.H., Parratt, J.D., Prineas, J.W., 2009. Multiple sclerosis: distribution of inflammatory cells in newly forming lesions. Ann. Neurol. 66 (6), 739-753.

Hohlfeld, R., Londei, M., Massacesi, L., Salvetti, M., 1995. T-cell autoimmunity in multiple sclerosis. Immunol. Today 16 (6), 259-261.

JM., C., 1868. Histologie de la sclérose en plaques. 41. Gaz des Hop, Paris, pp. 554-566.

Jordan, E.K., McFarland, H.I., Lewis, B.K., Tresser, N., Gates, M.A., Johnson, M., et al., 1999. Serial MR imaging of experimental autoimmune encephalomyelitis induced by human white matter or by chimeric myelin-basic and proteolipid protein in the common marmoset. AJNR Am. J. Neuroradiol. 20 (6), 965-976.

Kap, Y.S., Laman, J.D., t Hart, B.A., 2010. Experimental autoimmune encephalomyelitis in the common marmoset, a bridge between rodent EAE and multiple sclerosis for immunotherapy development. J. NeuroImmune Pharmacol. 5 (2), 220-230.

Katz, D., Taubenberger, J.K., Cannella, B., McFarlin, D.E., Raine, C.S., McFarland, H.F., 1993 Correlation between magnetic resonance imaging findings and lesion developmen in chronic, active multiple sclerosis. Ann. Neurol. 34 (5), 661-669.

Lassmann, H., 2011. Review: the architecture of inflammatory demyelinating lesions: implications for studies on pathogenesis. Neuropathol. Appl. Neurobiol. 37 (7) 698-710.

Lassmann, H., Bruck, W., Lucchinetti, C.F., 2007. The immunopathology of multiple sclerosis: an overview. Brain Pathol. 17 (2), 210-218.

Lathrop, S.K., Bloom, S.M., Rao, S.M., Nutsch, K., Lio, C.W., Santacruz, N., et al., 2011. Peripheral education of the immune system by colonic commensal microbiota. Nature 478 (7368), 250-254.

Leibovitch, E., Wohler, J.E., Cummings Macri, S.M., Motanic, K., Harberts, E., Gaitan, M.I., et al., 2013. Novel marmoset (Callithrix jacchus) model of human Herpesvirus 6A and 6B infections: immunologic, virologic and radiologic characterization. PLoS Pathog. 9 (1) e1003138.

Lucchinetti, C.F., Popescu, B.F., Bunyan, R.F., Moll, N.M., Roemer, S.F., Lassmann, H., et al., 2011. Inflammatory cortical demyelination in early multiple sclerosis. N. Engl. J. Med. 365 (23), 2188-2197.

Maggi, P., Macri, S.M., Gaitan, M.I., Leibovitch, E., Wholer, J.E., Knight, H.L., et al., 2014a. The formation of inflammatory demyelinated lesions in cerebral white matter. Ann. Neurol. 76 (4), 594-608. 
Maggi, P.M., N., L., Moretti, M., Grammatico, M., Chiti, S., Massacesi, L., 2014b. SWI enhances vein detection using gadolinium in multiple sclerosis. Acta Radiol. Short Rep. (Accepted for publication on October 31, 2014).

Marik, C., Felts, P.A., Bauer, J., Lassmann, H., Smith, K.J., 2007. Lesion genesis in a subset of patients with multiple sclerosis: a role for innate immunity? Brain 130 (Pt 11), 2800-2815

Massacesi, L., Genain, C.P., Lee-Parritz, D., Letvin, N.L., Canfield, D., Hauser, S.L., 1995. Active and passively induced experimental autoimmune encephalomyelitis in common marmosets: a new model for multiple sclerosis. Ann. Neurol. 37 (4), 519-530.

Polman, C.H., Reingold, S.C., Banwell, B., Clanet, M., Cohen, J.A., Filippi, M., et al., 2011. Diagnostic criteria for multiple sclerosis: 2010 revisions to the McDonald criteria. Ann. Neurol. 69 (2), 292-302.

Pomeroy, I.M., Matthews, P.M., Frank, J.A., Jordan, E.K., Esiri, M.M., 2005. Demyelinated neocortical lesions in marmoset autoimmune encephalomyelitis mimic those in multiple sclerosis. Brain 128 (Pt 11), 2713-2721.

Prineas, J.W., Parratt, J.D., 2012. Oligodendrocytes and the early multiple sclerosis lesion. Ann. Neurol. 72 (1), 18-31.

Richards, T.L., Alvord Jr., E.C., He, Y., Petersen, K., Peterson, J., Cosgrove, S., et al., 1995. Experimental allergic encephalomyelitis in non-human primates: diffusion imaging of acute and chronic brain lesions. Mult. Scler. 1 (2), 109-117.

Ryu, J.K., Petersen, M.A., Murray, S.G., Baeten, K.M., Meyer-Franke, A., Chan, J.P., et al., 2015. Blood Coagulation Protein Fibrinogen Promotes Autoimmunity and Demyelination via Chemokine Release and Antigen Presentation. 6 p. 8164.

Sati, P., Thomasson, D., Li, N., Pham, D., Biassou, N., Reich, D., et al., 2014. Rapid, highresolution, whole-brain, susceptibility-based MRI of multiple sclerosis. Mult. Scler.

Soon, D., Tozer, D.J., Altmann, D.R., Tofts, P.S., Miller, D.H., 2007. Quantification of subtle blood-brain barrier disruption in non-enhancing lesions in multiple sclerosis: a study of disease and lesion subtypes. Mult. Scler. 13 (7), 884-894.
Sriram, S., Steiner, I., 2005. Experimental allergic encephalomyelitis: a misleading model of multiple sclerosis. Ann. Neurol. 58 (6), 939-945.

Steinman, L., Zamvil, S.S., 2006. How to successfully apply animal studies in experimental allergic encephalomyelitis to research on multiple sclerosis. Ann. Neurol. 60 (1), $12-21$.

t Hart, B.A., Massacesi, L., 2009. Clinical, pathological, and immunologic aspects of the multiple sclerosis model in common marmosets (Callithrix jacchus). J. Neuropathol. Exp. Neurol. 68 (4), 341-355.

t Hart, B.A., van Meurs, M., Brok, H.P., Massacesi, L., Bauer, J., Boon, L., et al., 2000. A new primate model for multiple sclerosis in the common marmoset. Immunol. Today 21 (6), 290-297.

t Hart, B.A., Vogels, J., Bauer, J., Brok, H.P., Blezer, E., 2004. Non-invasive measurement of brain damage in a primate model of multiple sclerosis. Trends Mol. Med. 10 (2), 85-91.

tHart, B.A., van Kooyk, Y., Geurts, J.J., Gran, B., 2015. The primate autoimmune encephalomyelitis model; a bridge between mouse and man. Ann. Clin. Transl. Neurol. 2 (5), 581-593.

Wuerfel, J., Bellmann-Strobl, J., Brunecker, P., Aktas, O., McFarland, H., Villringer, A., et al., 2004. Changes in cerebral perfusion precede plaque formation in multiple sclerosis: a longitudinal perfusion MRI study. Brain 127 (Pt 1), 111-119.

Yednock, T.A., Cannon, C., Fritz, L.C., Sanchez-Madrid, F., Steinman, L., Karin, N., 1992. Prevention of experimental autoimmune encephalomyelitis by antibodies against alpha 4 beta 1 integrin. Nature 356 (6364), 63-66. 